\title{
Intrinsic energy partition in fission
}

\author{
M. Mirea ${ }^{1, a}$
}

Horia Hulubei National Institute for Physics and Nuclear Engineering, P.O. BOx MG-6, Bucharest, Romania

\begin{abstract}
The intrinsic energy partition between two complementary fission fragments is investigated microscopically. The intrinsic excitation energy of fission fragments is dynamically evaluated in terms of the time-dependent pairing equations. These equations are corroborated with two conditions. One of them fixes the number of particles and the other separates the pairing active spaces associated to the two fragments in the vicinity of the scission configuration. The excitation energy in a wide distribution of fission fragments is calculated for the ${ }^{234} \mathrm{U}$ parent nucleus.
\end{abstract}

\section{Introduction}

Under the action of a mutual Coulomb repulsion, at scission the fission fragments are accelerated in opposite directions. These fragments are highly excited. The maximal kinetic energy issued in the process amounts to the $Q$-value in the case of cold fission. The fragments decay on their ground states mainly by evaporation of neutrons and by radiation emission. It is known that the motion of any physical system is governed by conservative forces and by frictional ones that give rise to dissipation. Consequently, the excitation energy of the fragments must depend on the dynamics of the nuclear system in its path to scission. In this contribution the intrinsic energy partition between two complementary fission fragments is investigated microscopically in terms of the time-dependent pairing equations $[1,2]$. These equations are corroborated with two conditions. One of them fixes the number of particles and the other separates the pairing active spaces associated to the two fragments in the vicinity of the scission configuration. The fission path is obtained in the frame of the macroscopic-microscopic model. The single-particle level schemes are obtained within the two-center Woods-Saxon shell model. It is shown that the available intrinsic dissipated energy is not shared proportionally to the masses of the two fission fragments. If the heavy fragment possesses nucleon numbers close to the magic ones, the accumulated intrinsic excitation energy is lower than that of the light fragment.

Recently, this problem was intensively investigated within a wide range of methods, including Hartree-Fock theories [3], the statistical mechanics [4,5], the phenomenological point by point model [6,7], empirical evaluations [8], or the shell model in the sudden approximation [9].

\section{Model}

First of all, the main steps in deriving the microscopic equations of motion constrained by the projections of number of particles are presented. The starting point of the model is the energy functional

$$
\mathcal{L}=\left\langle\varphi\left|H-i \hbar \frac{\partial}{\partial t}-\lambda\left(N_{1} \hat{N}_{2}-N_{2} \hat{N}_{1}\right)\right| \varphi\right\rangle
$$

\footnotetext{
a e-mail: mirea@ifin.nipne.ro
} 
where

$$
H(t)=\sum_{k>0} \epsilon_{k}\left(a_{k}^{+} a_{k}+a_{\bar{k}}^{+} a_{\bar{k}}\right)-G \sum_{k, i>0} a_{k}^{+} a_{\bar{k}}^{+} a_{i} a_{\bar{i}}
$$

is the Hamiltonian with pairing residual interactions, $\epsilon_{k}$ being single particle energies,

$$
|\varphi\rangle=\sum_{k}\left(u_{k}+v_{k} a_{k}^{+} a_{\bar{k}}^{+}\right)|0\rangle
$$

is the Bogoliubov wave function with BCS occupation $v_{k}$ and vacancy $u_{k}$ amplitudes. $\lambda$ represents a Lagrange multiplier and

$$
\begin{aligned}
& \hat{N}_{1}=\sum_{k_{1}}\left(a_{k_{1}} a_{k_{1}}^{+}+a_{\bar{k}_{1}} a_{\bar{k}_{1}}^{+}\right), \\
& \hat{N}_{2}=\sum_{k_{2}}\left(a_{k_{2}} a_{k_{2}}^{+}+a_{\bar{k}_{2}} a_{\bar{k}_{2}}^{+}\right)
\end{aligned}
$$

are operators for the number of particles in the pairing active level space pertaining to the nuclei $A_{1}$ and $A_{2}$, respectively. The summation index $k, k_{1}$ and $k_{2}$ run over all states considered in the pairing active space, the states of the fragment one, and the those of the fragments two in the same space, respectively. $N_{1}$ and $N_{2}$ are the correspondent number of particles belonging to the two fission fragments. Obviously, the following identity

$$
\left\langle\varphi\left|\left(N_{1} \hat{N}_{2}-N_{2} \hat{N}_{1}\right)\right| \varphi\right\rangle=0
$$

can be used to fix the number of particle $N_{1}$ and $N_{2}$ in the two fission fragments. The condition (5) is added to the energy functional (1) by mean of the Lagrange multiplier as an implicit condition. Therefore, the equation (1) is subject to a supplementary condition that project the number of particles onto the two fragments. The next equations are obtained for the TDPE:

$$
\begin{array}{r}
i \hbar \dot{\rho}_{k_{1}}=\kappa_{k_{1}} \Delta_{1}^{*}-\kappa_{k_{1}}^{*} \Delta_{1}, \\
i \hbar \dot{\rho}_{k_{2}}=\kappa_{k_{2}} \Delta_{2}^{*}-\kappa_{k_{2}}^{*} \Delta_{2}, \\
i \hbar \dot{\kappa}_{k_{1}}=\left(2 \rho_{k_{1}}-1\right) \Delta_{1}-2 \kappa_{k_{1}}\left(\epsilon_{k_{1}}+\lambda N_{2}\right), \\
i \hbar \dot{\kappa}_{k_{2}}=\left(2 \rho_{k_{2}}-1\right) \Delta_{2}-2 \kappa_{k_{2}}\left(\epsilon_{k_{2}}-\lambda N_{1}\right)
\end{array}
$$

where $\rho_{k}=\left|v_{k}\right|^{2}$ are occupation probabilities, $\kappa_{k}=u_{k}^{*} v_{k}$ are pairing moment components, and $\Delta=$ $G \sum_{k} \kappa_{k}$ is the pairing gap. $\Delta_{1}=G_{1} \sum_{k_{1}} \kappa_{k_{1}}+G_{12} \sum_{k_{2}} \kappa_{k_{2}}$ and $\Delta_{2}=G_{12} \sum_{k_{1}} \kappa_{k_{1}}+G_{2} \sum_{k_{2}} \kappa_{k_{2}}$ are the gaps for the two fragments. It must be noticed that if $G_{12}=0$, then we obtain two sets of non coupled equations, one set for each fission fragment [1]. This last condition separates the pairing active space into two sub-spaces. A particular form of these equations was deduced for the first time in Refs. [10, 11]. They were developed in the last years [12] to take into account the Landau-Zener effect [13]. the pair breaking mechanism [14], and the dissipation in the effective mass [15].

These TDPE are solutions of an optimization problem and can offer a measure of the average dissipated energy at a given deformation during the disintegration process provided that the velocity of the deformations are known. The difference between the total energy value $E$ obtained within the TDPE and $E_{0}$ given by the static BCS-equations represents an approximate measure for the dissipation $E^{*}$ :

$$
E^{*}=E-E_{0} \text {. }
$$

$E$ is expressed simply in terms of $\rho_{k}$ and $\kappa_{k}$

$$
E=2 \sum_{k} \epsilon_{k} \rho_{k}-G\left|\sum_{k} \kappa_{k}\right|^{2}-G \sum_{k} \rho_{k}^{2},
$$

and $E_{0}$ corresponds to the values $\rho_{k}^{0}$ and $\kappa_{k}^{0}$ associated to the lower energy state, that is, obtained from BCS equations. This definition was introduced in Ref. [10] where the nuclear viscosity coefficient is determined by comparing microscopic results with hydrodynamic ones. So, the TDPE provide the values of $\rho_{k}$ and $\kappa_{k}$ in each fragment. By calculating the BCS solutions of the same fragment in the average deformation, in the corresponding pairing active space, the intrinsic excitation is obtained with formula (7). 


\section{Results}

The low energy fission of ${ }^{234} \mathrm{U}$ will be investigated. In the Macroscopic-Microscopic Method (MMM), the whole system is characterized by some collective coordinates that vary in time leading to a split of the nucleus into two fragments of masses $A_{1}$ and $A_{2}$. The macroscopic-microscopic model provides a single particle potential in which the nucleons move independently. As mentioned in Ref. [10], such a description is within the spirit of the more rigorous Hartree-Fock method which defines the potential in terms of the instantaneous positions of the nucleons. The basic ingredient of the MMM is the nuclear shape parametrization. An axial symmetric nuclear shape is obtained by smoothly joining two spheroids of semi-axis $a_{i}$ and $b_{i}(i=1,2)$ with a neck surface generated by the rotation of an arc of circle. By imposing the condition of volume conservation we are left with five independent generalized coordinates that are associated to five degrees of freedom: the elongation $R$ denoting the distance between the centers of the fragments; the necking parameter $C=S / R_{3}$ related to the curvature of the neck; the eccentricities $\epsilon_{i}=\left[1-\left(b_{i} / a_{i}\right)^{2}\right]^{1 / 2}$ if $a_{i} \geq b_{i}$ (or $\epsilon_{i}=-\left[1-\left(a_{i} / b_{i}\right)^{2}\right]^{1 / 2}$ if $\left.a_{i}<b_{i}\right)$ associated to deformations of the two fragments $\left(a_{i}\right.$ and $b_{i}$ denoting the semi-axis) and the mass asymmetry parameter $\eta=a_{1} / a_{2}$ given by the ratio of the major semi-axis. This parametrization was widely used previously in the calculation concerning cluster decay [17-20] or barriers for fission [21-23]. A Woods-Saxon two center shell model [13] compatible with this nuclear shape parametrization is used to compute the single particle levels and to disentangle the wave functions before that the scission is reached [1]. Using the minimal action principle a trajectory in the configuration space beginning with the parent ground state and ending in the exit point of the barrier is determined. The fission barrier along the minimal action path is presented in Fig. 1. A method developped in Ref. [21] is used to find the barriers associated to the most favorable partitions in the mass distribution. These partitions are selected from the maximal yields given in Ref. [24].

The ingredients needed to obtain the dissipated energy are the single particle energies and the internuclear velocity. These energies are calculated within the Woods-Saxon two center model and the internuclear is considered to be $10^{6} \mathrm{~m} / \mathrm{s}$. This velocity gives a tunneling time of $10^{-21} \mathrm{~s}$. A similar value resorts in calculations devoted to alpha [25] and cluster decays [26-28]. This internuclear velocity is in agreement with the results obtained in Ref. [29,30]. In the previous reference a parent metastable state is generated and a tunneling time of the order of $\left(10^{-21}-10^{-22}\right) \mathrm{s}$ is computed.

It is not possible to extract directly the energy partition from experimental data. Nevertheless, the main de-excitation process is the neutron evaporation. Therefore, indirect information can be obtained from neutron multiplicities, for which accurate results are available [31]. The excitation energy of each fragment is computed within the relation (7). The results are plotted in Fig. 2. Several experimental features are reproduced by the theoretical data. The deeply minimum in the neutron multiplicity occurs close to the mass of the doubly magic nucleus ${ }^{132} \mathrm{Sn}$. A maximal value of the neutron multiplicity is obtained for the mass 116, that is in the symmetric fission region. In general, the excitation energy of the light fragment is larger than that of the heavy one.

We can compare also the behavior of the average neutron multiplicity with the sum of total excitation energies of partners. The average neutron multiplicity is defined as the sum of neutrons from both fragments [31]. The minimal value of the average neutron multiplicity appears around $130 \mathrm{u}$. This fact is reproduced by theoretical data. Our results plotted with a thick dashed line in Fig. 2 give a minimum of the excitation. An enhanced neutron emission is obtained in the symmetric fission and the very asymmetric region. Between these extreme, the total neutron emission shows a structure with a maximal value around $A_{1}=140$. These behavior are also exhibited by the total excitation energy [31]. From another respect, it must be mentioned that the excitation energy exhibits a structure with strong fluctuations, especially for $A_{2}<82$. A possible explanation for this peculiar aspect could be related to the existence of another fission path associated to large mass asymmetries. Therefore, the deformations and the dynamics assumed in this region could be not realistic.

Excepting the strong fluctuations related to the large mass asymmetries, the results agree qualitatively well with the data that correspond to neutron multiplicities. It is a first microscopic description of the intrinsic energy partition in a wide range of fission channels that succeed to reproduce the main behavior of the neutron multiplicities. 


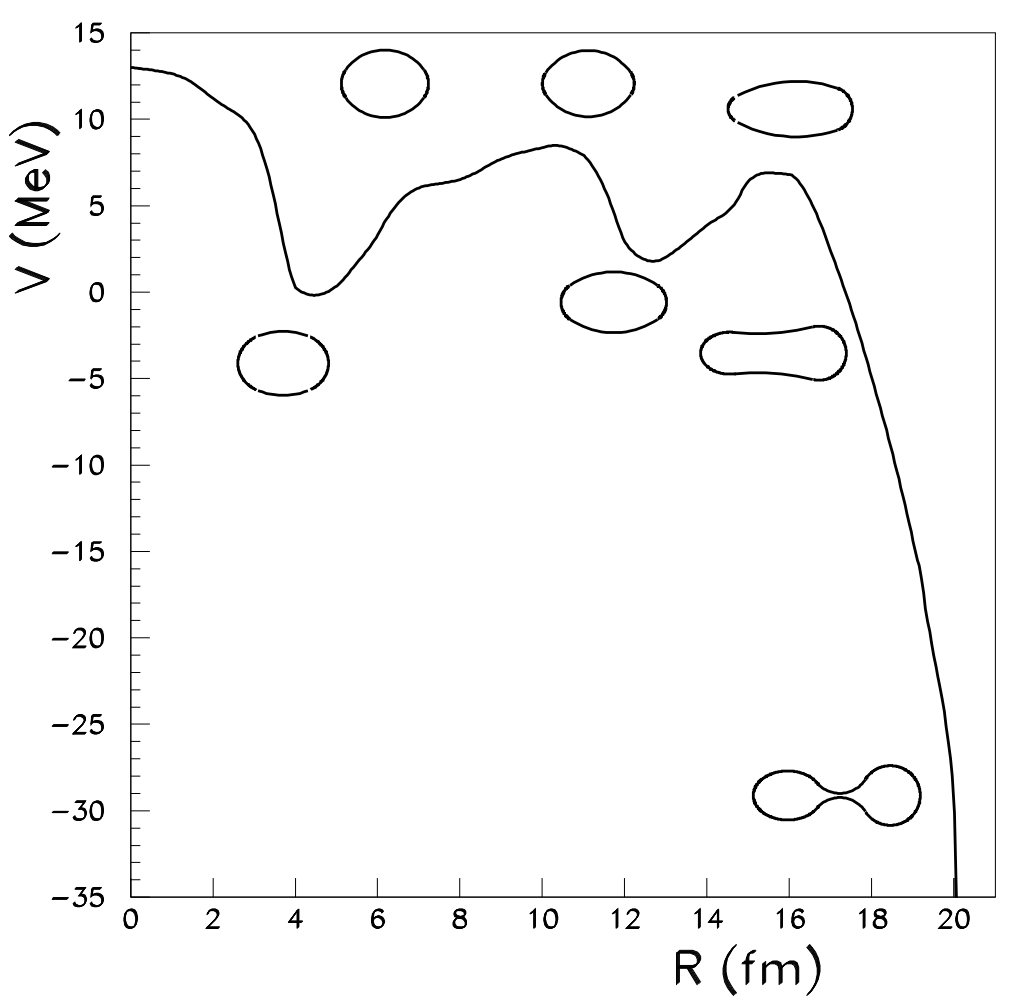

Fig. 1. ${ }^{234} \mathrm{U}$ fission barrier $V$ for a final partition ${ }^{102} \mathrm{Zr}+{ }^{132} \mathrm{Te}$ determined along the minimal action trajectory. Some particular shapes related to the ground state, the extremes of the barrier, the exit point and the scission point are inserted in the plot. The distances for the elongation $R$ that characterizes the shapes are 4.17, 7.7, 10.43, 12.64, $15.53,17.53$ and $20.2 \mathrm{fm}$.

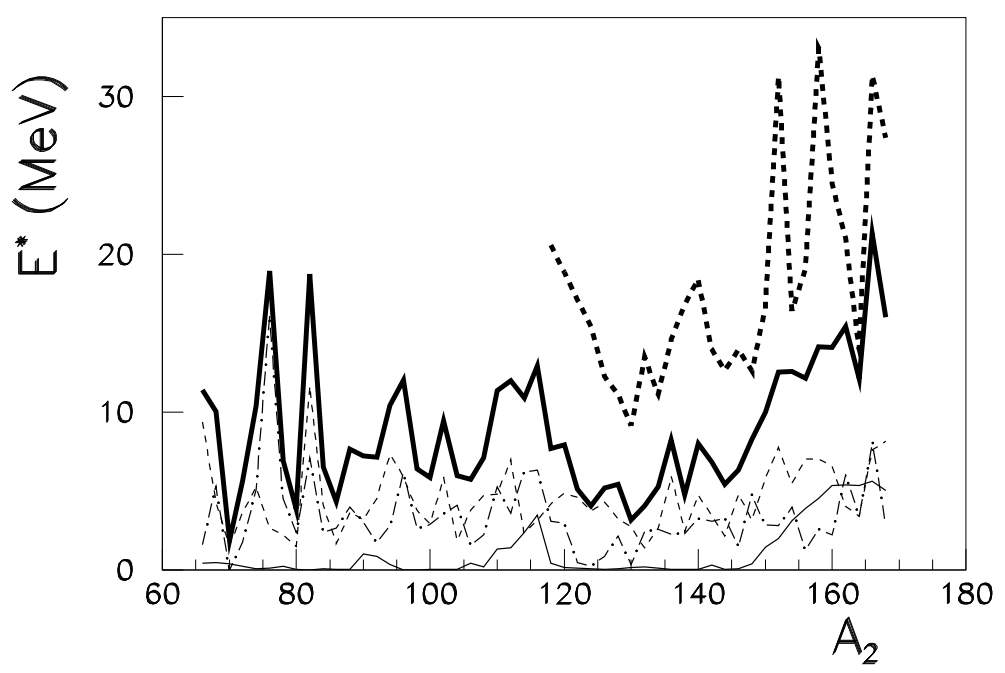

Fig. 2. The total excitation energies $E^{*}$ of the fission fragments are plotted versus their masses with a thick full line. A thin full line is used for the deformation energy, a thin dashed line gives the neutron dissipated energy while the thin dot dashed line gives the dissipated energy for the proton space. The sum of excitation energies of the fission partners is plotted as function of the heavy fragment mass with a thick dashed line. 


\section{WONDER-2012}

This work was supported by CNCS-UEFISCDI, project number PN-II-ID-PCE-2011-3-0068.

\section{References}

1. M. Mirea, Phys. Rev. C 83, (2011) 054608

2. M. Mirea, Phys. Lett. B doi:10.1016/j.physletb.2012.09.023

3. W. Younes and D. Gogny, Phys. Rev. Lett. 107, (2011) 132501

4. K.-H. Schmidt and B. Jurado, Phys. Rev. Lett. 104, (2010) 212501

5. K.-H. Schmidt and B. Jurado, Phys. Rev. C 83, (2011) 014607

6. C. Morariu, A. Tudora, F.J. Hambsch, S. Oberstedt and C. Manailescu, J. Phys. G 39, (2012) 055103

7. C. Manailescu, A. Tudora, F.-J. Hambsch, C. Morariu, S. Oberstedt, Nucl. Phys. A 867, (2011) 12

8. Chen Yong-Jing, Qian Jing, Liu Ting-Jin, Li Zhu-Xia, Wu Xi-Zhen and Shu Neng-Chuan, Int. J. Mod. Phys. E 21, (2012) 1250073

9. N. Carjan, F.-J. Hambsch, M. Rizea and O. Serot, Phys. Rev. C 85, (2012) 044601

10. S.E. Koonin and J.R. Nix, Phys. Rev. C 13, (1976) 209

11. J. Blocki and H. Flocard, Nucl. Phys. A 273, (1976) 45.

12. M. Mirea, Int. J. Mod. Phys. E 21, (2012) 1250035

13. M. Mirea, Phys. Rev. C 78, (2008) 044618

14. M. Mirea, Phys. Lett. B 680, (2009) 316

15. M. Mirea, Rom. Rep. Phys. 63, (2011) 676

16. M. Mirea and R.C. Bobulescu, J. Phys. G 37, (2010) 055106

17. M. Mirea, A. Sandulescu, and D.S. Delion, Nucl. Phys. A 870-871 (2011) 23.

18. M. Mirea, A. Sandulescu, and D.S. Delion, Europ. Phys. J. A 48 (2012) 86.

19. M. Mirea, A. Sandulescu, and D.S. Delion, Proc. Rom. Acad. Series A 12, (2011) 203

20. M. Mirea, Rom. J. Phys. 57, (2012) 372

21. M. Mirea, D.S. Delion and A. Sandulescu, Phys. Rev. C 81 (2010) 044317.

22. M. Mirea and L. Tassan-Got, Cent. Europ. J. Phys. 9, (2011) 116

23. I. Companis, M. Mirea and A. Isbasescu, Rom. J. Phys. 56, (2011) 63

24. A.C. Wahl, At. Data Nucl. Data Tables 39, (1988) 1.

25. M. Mirea, Phys. Rev. C 63, (2001) 034603

26. M. Mirea, Phys. Rev. C 57, (1998) 2484

27. M. Mirea, Eur. Phys. J. A 4, (1999) 335

28. M. Mirea, Mod. Phys. Lett. A 18, (2003) 1809

29. O. Serot, N. Carjan and D. Strottman, Nucl. Phys. A 569, (1994) 562

30. N. Carjan, O. Serot and D. Strottman, Z. Phys. A 349, (1994) 353

31. K. Nishio, M. Nakashima, I. Kimura and Y. Nakagome, J. Nucl. Sci. and Tech. 35, (1998) 631 